

\title{
A Rio+20 e o quadro institucional pelo desenvolvimento sustentável: o papel dos governos subnacionais na governança ambiental global ${ }^{*}$
}

\author{
$R I O+20$ and institutional framework \\ for sustainable development: the role \\ of subnational governments in global \\ environmental governance
}

Fernando Rei ${ }^{1}$ Joana Setzer ${ }^{2}$ Kamyla Borges Cunha ${ }^{3}$

\section{Resumo}

Este artigo tem por objetivo examinar o papel dos governos subnacionais na construção da governança ambiental global. Com esse fim, considera os fatores que fazem dos governos subnacionais atores relevantes na promoção do desenvolvimento sustentável e analisa a maneira pela qual esses atores são incluídos pelo "Esboço zero" (Zero Draft) do documento final da Rio+20 no quadro institucional do desenvolvimento sustentável. Tendo por base a emergência dos governos subnacionais e suas redes horizontais, o artigo examina as atividades da Rede de Governos Regionais para o Desenvolvimento Sustentável (nrg4SD) desde sua criação, dez anos atrás, até seu posicionamento sobre o Zero Draft. O artigo sugere que a paradiplomacia ambiental pode desempenhar um papel cada vez mais relevante no quadro institucional que será necessário para o alcance de um desenvolvimento sustentável. Em primeiro lugar, porque os problemas que afetam o mundo hoje exigem uma resposta de múltiplos atores, em múltiplas escalas, cabendo aos governos subnacionais o papel crucial de desenvolver e implementar as políticas necessárias à promoção do paradigma da sustentabilidade mais próximos do cidadão, e em segundo lugar, porque, para se alcançar uma verdadeira governança ambiental global, os governos subnacionais precisam ser reconhecidos como stakeholders governamentais no conjunto de atores que integra o cenário internacional de tomada de decisão.

Palavras-chave: Paradiplomacia ambiental. Mudanças climáticas. Governos subnacionais. Nrg4SD. Rio+20.

\begin{abstract}
This article examines subnational government's role in global environmental governance. With such an end, the article considers the characteristics that make subnational governments relevant actors in the promotion of sustainable development and the ways in which the Zero Draft of the outcome document of Rio+20 engages these actors in the institutional framework for sustainable development. Considering the emergence of subnational governments and their horizontal networks in global environmental governance, the article examines the activities of
\end{abstract}

Artigo recebido em 17/03/2012

Artigo aprovado em 08/06/2012

1 Professor do Programa de Doutorado em Direito Ambiental Internacional. Diretor Científico de SBDIMA (Sociedade Brasileira de Direito Internacional Ambiental). Email: fernandorei@ ig.com.br.

2 Grantham Institute on Climate Change and the Environment. Email: j.setzer@lse.ac.uk.

3 Professora de Legislação Ambiental. Email: kamilaborges@gmail.com. 
the Network of Regional Governments for Sustainable Development (nrg4SD), since its creation, ten years ago, to its position in relation to the Zero Draft. The article suggests that environmental paradiplomacy can play an increasingly relevant role in the institutional framework that will be needed for sustainable development. First because the problems that affect the world in the present require answers from multiple actors, in multiple scales, having subnational governments the crucial role of developing and implementing policies at citizen's level. Second, because in order to achieve global environmental governance, subnational governments must be recognised as governmental stakeholders among the actors responsible for the international decision-making process.

Keywords: Environmental paradiplomacy. Climate change. Subnational governments. Nrg4SD. Rio+20.

\section{Introdução}

A Conferência das Nações Unidas sobre Desenvolvimento Sustentável (Rio+20) marca os vinte anos de realização da Conferência das Nações Unidas sobre Meio Ambiente e Desenvolvimento (Rio-92) e os dez anos da Cúpula Mundial sobre Desenvolvimento Sustentável em Johanesburgo. A Rio+20 marca também o aniversário de 10 anos de criação da Rede de Governos Regionais para o Desenvolvimento Sustentável (nrg4SD). A nrg4SD foi a primeira rede transnacional de governos subnacionais a tratar do tema do desenvolvimento sustentável em uma escala global. ${ }^{4}$ Esta rede conta com a participação de 36 governos subnacionais de 18 países e 7 associações de governos regionais, que ao todo representam mais de 1000 governos em todo o mundo. ${ }^{5}$

A criação de redes transnacionais de governos subnacionais como a nrg4SD sinaliza a contribuição dos governos subnacionais às novas dinâmicas do Direito Internacional do Meio Ambiente (DIMA), fenômeno que

${ }^{4}$ HAPPAERTS, Sander; VAN DEN BRANDE, Karoline; BRUYNINCKX, Hans. Governance for Sustainable Development at the Inter-subnational Level: The Case of the Network of Regional Governments for Sustainable Development (nrg4SD). Regional \& Federal Studies, v. 20, n. 1, p. 127-149, 2010 . recebe o nome de "paradiplomacia ambiental". Este artigo sugere que a paradiplomacia ambiental pode desempenhar um papel cada vez mais relevante no quadro institucional que será necessário para o alcance de um desenvolvimento sustentável. Em primeiro lugar, porque os problemas que afetam o mundo hoje exigem uma resposta de múltiplos atores, em múltiplas escalas, cabendo aos governos subnacionais o papel crucial de desenvolver e implementar as políticas necessárias à promoção do paradigma da sustentabilidade mais próximos do cidadão. Em segundo lugar, porque, para se alcançar uma verdadeira governança ambiental global, os governos subnacionais precisam ser reconhecidos como atores transnacionais, ou seja, como stakeholders governamentais no conjunto de atores que integra o cenário internacional de tomada de decisão.

Com este fim, o presente artigo apresenta três seções. A primeira discorre sobre a importância dos governos subnacionais na promoção do desenvolvimento sustentável. A segunda parte examina a emergência dos governos subnacionais e suas redes horizontais na construção da governança ambiental global; a nrg4SD ilustra esse processo e será utilizada como estudo de caso. A terceira parte do artigo conclui que a paradiplomacia ambiental, ou seja, a inserção dos governos subnacionais no DIMA deve ser considerada no quadro institucional do desenvolvimento sustentável.

\section{A importância dos governos subnacionais na promoção do desenvolvimento sustentável}

Esta Seção demonstra a importância dos governos subnacionais na promoção do desenvolvimento sustentável. Sugere-se (item 1.1.) que existem ao menos quatro motivos pelos quais os governos subnacionais devem ser reconhecidos como centrais para a promoção do desenvolvimento sustentável: (i) os problemas ambientais sobre os quais se depara a humanidade devem ser encarados de forma coordenada; (ii) as causas e as consequências desses problemas ocorrem e são sentidas concretamente nos níveis locais e regionais; (iii) muitas vezes, cabe aos governos subnacionais a definição e a implementação de políticas públicas que visem ou levem em conta o desenvolvimento sustentável; e (iv) não existe mais uma exclusividade da via internacional tradicional para o enfrentamento dos problemas globais. Ao que tudo indica, 
esse papel crucial desempenhado pelos governos subnacionais no desenvolvimento sustentável será reconhecido pelo documento final da Rio+20. A abordagem que consta do "Esboço Zero" (Zero Draft) do documento final da Conferência será objeto de análise no item 1.2, e depois novamente, no item 2.3, no que tange às contribuições que estão sendo sugeridas pela nrg4SD.

\subsection{Por que os governos subnacionais são centrais para o desenvolvimento sustentável?}

A solução dos problemas ambientais globais requer a construção de uma governança global, por meio da qual as diferentes formas e níveis de enfrentamento dos problemas passam a coexistir numa dinâmica de complementaridade. Assim, o avanço dos regimes internacionais baseados em tratados multilateralmente firmados é fortalecido e fomentado pelas iniciativas desenvolvidas nos níveis infra e transnacionais e por atores ainda não formalmente parte do sistema jurídico internacional. Há, portanto, uma dinâmica de mútua influência, da qual o DIMA se nutre. Por que então se faz necessária a participação dos governos subnacionais nesse processo de governança global?

Em primeiro lugar, a escala e a urgência dos desafios com os quais a humanidade se depara hoje exige uma resposta coordenada, envolvendo múltiplos atores em todos os níveis de governo. O mundo hoje é mais interdependente que no passado. Os problemas ambientais, assim como os atinentes aos direitos humanos, finanças e comércio, somente podem encontrar soluções satisfatórias se negociadas e regulamentadas pelo conjunto dos Estados e demais atores transnacionais, dinâmica essa observada nas negociações multilaterais. Nessa linha, Krahmann ${ }^{6}$ destaca que a governança compreende processos e estruturas que permitem a atores governamentais e não governamentais coordenar suas necessidades e interesses interdependentes por intermédio da construção e implantação de políticas. Para Hoffmann, ${ }^{7}$ em uma governança global, as regras devem ser feitas acima, abaixo e entre as autoridades estabelecidas.

6 KRAHMAN, Elke. National, regional and global governance: one phenomenon or many. Global governance, v. 9, p. 323346, 2003.

7 HOFFMANN, Matthew J. Climate governance at the crossroads. New York: Oxford University Press, 2011.
Em segundo lugar, tanto as causas como as consequências dos problemas ambientais ocorrem e são sentidos concretamente nos níveis locais e regionais, forçando os atores subnacionais a uma resposta mais proativa e efetiva. ${ }^{8},{ }^{910},{ }^{11}$ Tomando-se o exemplo do problema das mudanças climáticas, fica claro como seus impactos ignoram barreiras estatais. De fato, o equilíbrio climático constitui um continuum ecológico que se projeta tanto nos espaços submetidos à soberania dos Estados, como mais além deles, ${ }^{12}$, e ainda porque os impactos desse problema ambiental são ao fim e ao cabo sentidos concretamente nos níveis infranacionais. ${ }^{13}$ Conforme salientado por Liftin, ${ }^{14}$ a dualidade global-local das mudanças climáticas inculca o sentido de responsabilidade por seu enfrentamento em todos os níveis de organização social.

Em terceiro lugar, a maioria dos governos subnacionais tem competências legislativas e administrativas sobre matéria ambiental e políticas sociais, cabendo, portanto, a esses governos a definição de políticas públicas que visem ou levem em conta o desenvolvimento sustentável. Soma-se a esse ponto o fato de que as iniciativas dos governos subnacionais no combate às mudanças climáticas globais têm ampliado os resultados da governança ambiental. Como asseveram Okereke et al, ${ }^{15}$ em alguns casos, essas iniciativas somam-se aos esforços nacionais e internacionais de enfrentamento do problema, contri-

8 BULKELEY, Harriet; BETSILL, Michelle. Cities and climate change: urban sustainability and global environmental governance. London: Routledge, 2003.

9 RABE, Barry. Statehouse and greenhouse: the emerging politics of american climate change policy. Washington, D.C.: Brookings Institution Press, 2004.

${ }^{10}$ RABE, Barry. States on steroids: the intergovernmental odyssey of american climate policy. Review of policy research, v. 25, n. 2, p. 105-128, 2008 .

${ }^{11}$ SETZER, Joana. Governança multinível das mudanças climáticas: políticas subnacionais e ações transnacionais em São Paulo. In: REI, Fernando Cardozo Fernandes (Org.). Direito e desenvolvimento: uma abordagem sustentável. No prelo.

${ }^{12}$ RUIZ, Jose Juste. Derecho Internacional del medio ambiente. Madrid: McGraw Hill, 1999. p. 479.

${ }^{13}$ BODANSKY, Daniel. The legitimacy of international governance: a coming challenge for international environmental law? The American Journal of International Law, v. 93, n. 3, p. 596-624, jul.1999.

${ }^{14}$ LITFIN, Karen. Environment, Wealth and Authority: global climate change and emerging modes of legitimation. International Studies Review, v. 2, n. 2, p. 119-148, 2000.

${ }^{15}$ OKEREKE, Chukwumerije; BULKELEY, Harriet; SCHROEDER, Heike. Conceptualizing climate governance beyond the international regime. Global Environmental Politics, v. 9, n. 1, p. 58-68, fev. 2009. 
buindo para que os Estados cumpram com compromissos internacionalmente assumidos. Em outros casos, as ações subnacionais resultam em efetivas reduções de gases de efeito estufa (GEE), apesar da inércia dos governos nacionais. De qualquer forma, essas iniciativas acabam exercendo uma influência estratégica nas negociações internacionais do regime climático, uma vez que funcionam como elemento de pressão (e constrangimento) para que os Estados avancem em seus compromissos e estejam abertos a novos esforços multilaterais.

Em quarto lugar, não é mais possível defender um "retorno à realpolitik", ou seja, a ideia de que as políticas ambientais somente podem ser definidas por meio de um acordo consensual de todos os Estados participantes. ${ }^{16} \mathrm{~A}$ via internacional tradicional de enfrentamento dos problemas globais, por ser oficializada por meio de acordos firmados por consenso entre Estados soberanos, sofre a influência direta de interesses internos e externos, particularmente num contexto de crescente interdependência, sobretudo econômica. ${ }^{17} \mathrm{Em}$ geral, esses interesses não se coadunam com as exigências de enfrentamento dos problemas ambientais globais, posto que, enquanto têm um horizonte de curto prazo ou apresentam caráter predominantemente político-econômico, os problemas ambientais requerem ações de longo prazo e uma visão estrategicamente mais ampla. Assim, quanto mais distante a resposta dos Estados for de uma verdadeira cooperação e solidariedade para a solução dos problemas ambientais, mais questionável se torna seu grau de legitimidade e autoridade. $^{18}$

O caso das mudanças climáticas novamente ilustra bem esse argumento. Na esfera internacional, a resposta às mudanças climáticas tem sido construída por meio do regime climático, um conjunto de normas, instituições e medidas de mitigação das emissões de gases de efeito estufa e de adaptação aos impactos previstos, ancorados

${ }^{16}$ BERNSTEIN, Steven et al. A tale of two copenhagens: carbon markets and climate governance. Millennium: Journal of International Studies, v. 39, n.1, p. 161-173, 2010.

${ }^{17}$ LEIS, Hector; VIOLA, Eduardo. América del Sur en el mundo de las democracias de mercado. Rosario: Homo Sapiens Ediciones, 2008. p.196.

${ }^{18}$ REI, Fernando; CUNHA, Kamyla. Mudanças climáticas globais: desafio a uma nova relação entre o Direito Internacional do Meio Ambiente e as Relações Internacionais. In: CASELLA, Paulo Borba et al. (Org.). Direito internacional, humanismo e globalidade: guido Fernando Silva Soares Amicorum Discipulorum Líber. São Paulo: Atlas, 2008. p. 487-502. sob dois tratados: a Convenção Quadro das Nações Unidas sobre a Mudança do Clima (UNFCCC) e o Protocolo de Quioto. Mas apesar de ser considerado um marco do DIMA, ao se conferir demasiada importância às negociações entre Estados nacionais, deixa-se de ver os avanços que surgem em formas alternativas de governança. Nesse sentido, a crescente participação dos estados subnacionais no regime internacional do clima, ainda que de forma paralela à atuação dos estados nacionais, demonstra a ampliação tanto dos atores envolvidos nas negociações multilaterais como das escalas envolvidas no DIMA. ${ }^{19}$

Portanto, fica fácil reconhecer que o efetivo enfrentamento das questões ambientais globais necessita da ação cooperada e coordenada de sistemas de governança baseados em diversos níveis (estatais, supra, infra e interestatais) e compostos por atores estatais, infraestatais (regionais e locais) e não governamentais, cada um desempenhando uma variedade de papéis. ${ }^{20},{ }^{21},{ }^{22}$ Fala-se, nesse caso, de uma nova governança ambiental global. Nas palavras de Reed e Bruyneel:

[...] as environmental problems cross borders spatially (affecting multiple jurisdictions) and temporally (posing risks for the present and for future generations), they necessitate cooperation among nations and stakeholder groups in a form of global environmental governance. ${ }^{23}$

\section{2 reconhecimento pelo zero draft da Rio+20}

O "Esboço Zero" (Zero Draft) do documento final da Conferência contém as contribuições nacionais de todos os Estados-Membros como proposta inicial para o texto a ser adotado na Rio+20 e é acompanhado pela nrg4SD (mais a esse respeito, na próxima seção), reconhece o relevante papel desempenhado pelos governos locais no desenvolvimento sustentável e inclui a governança em to-

${ }^{19}$ OSOFSKY, Hari M. Multiscalar governance and climate change: reflections on the role of states and cities at Copenhagen. Maryland Journal of International Law, v. 25, n. 64, p. 64-85, 2010.

${ }^{20}$ BULKELEY, Harriet; BETSILL, Michelle. Cities and climate change: urban sustainability and global environmental governance. London: Routledge, 2003.

${ }^{21}$ BULKELEY, Harriet. Reconfiguring environmental governance: towards a politics of scales and networks. Political Geography, n. 24, p. 875-902, 2005.

${ }^{22}$ ADGER, Neil; JORDAN, Andrew (Eds.). Governing sustainability. Cambridge: Cambridge University Press, 2009.

${ }^{23}$ REED, Maureen; BRUYNEEL, Shannon. Rescaling environmental governance, rethinking the state: a three-dimensional review. Progress in Human Geography, v. 34, n. 5, p. 646-653, 2010. 
dos os níveis de governo dentro do quadro institucional do desenvolvimento sustentável. ${ }^{24}$

A Seção II do Zero Draft ("Renovando Compromissos Políticos"), no item C ("Envolvendo os Principais Grupos"), parágrafo 17 reconhece o papel dos principais grupos (mulheres, crianças e adolescentes, populações indígenas, ONGs, autoridades locais, trabalhadores e associações de classe, comércio e indústria, comunidade tecnológica e científica e agricultores) no desenvolvimento sustentável. Em seguida, o parágrafo 20, especificamente reconhece o papel essencial dos governos locais e a necessidade de integrá-los em todos os níveis de tomada de decisão do desenvolvimento sustentável. Na mesma Seção, item D, parágrafo 22, há o comprometimento de melhorar a governança e capacidades em todos os níveis - global, regional, nacional e local - para promover um processo de tomada de decisão integrado, para preencher deficiências na fase de implementação e promover coerência entre diferentes instituições.

Merece destaque a Seção IV ("Quadro institucional para um desenvolvimento sustentável”), a qual estabelece que, para fortalecer e integrar os três pilares do desenvolvimento sustentável, será preciso reconhecer a importância de uma governança nos níveis local, nacional, regional e global, mediante o estabelecimento de políticas públicas e o monitoramento da implementação da Agenda $21 \mathrm{em}$ todos esses níveis (item A, parágrafo 44). Em outras palavras, um dos principais aspectos do quadro institucional para o desenvolvimento sustentável diz respeito ao estabelecimento de políticas públicas por governos subnacionais. Esse quadro é reiterado no item $\mathrm{D}$, o qual requer o fortalecimento de mecanismos regionais e sub-regionais na promoção do desenvolvimento sustentável (parágrafo 60) e a coordenação entre políticas nacionais e locais (parágrafo 62).

\section{A emergência dos governos subnacionais e suas redes horizontais na construção da go- vernança ambiental global}

As duas primeiras décadas deste novo milênio já registram uma série de fatos sobre os quais a sociedade

\footnotetext{
${ }^{24}$ UN. The future we want: Zero Draft of the outcome document. Disponível em: <http://www.uncsd2012.org/rio20/futurewewant.html>. Acesso em: 11 mar. 2012.
}

internacional é chamada a tratar a partir da premissa de que as soluções deverão ser pensadas e implantadas de maneira integrada, solidária e multilateral. Sejam problemas do mercado financeiro, das ações terroristas, da emergência de processos democráticos no mundo árabe, dos grandes temas ambientais globais, trata-se de um conjunto de assuntos, direta ou indiretamente comuns a todos os Estados. De um lado, a estrutura do mundo contemporâneo é menos flexível e dinâmica do que a experimentada durante a década que seguiu a Rio-92. De outro lado, esse mundo pressupõe cooperação e interdependência, com o encaminhamento de soluções feito dentro de um modelo de governança.

Naturalmente essa nova problemática incide na estrutura e na dinâmica do Direito Internacional. $\mathrm{O}$ atual momento histórico reclama novas áreas do saber jurídico, a renovação das bases da ordem internacional, a reforma da lógica do sistema das Nações Unidas. Abordando a ideia, Gonçalves e Fontoura Costa apontam para o estabelecimento de novas relações entre os atores nos mais variados níveis, para que, em conjunto, negociando e construindo pactos e normas comuns, possam, de fato, enfrentar as situações e obstáculos comuns. ${ }^{25}$ Esta seção aborda a inserção desses novos atores e níveis e, especialmente, a emergência dos governos subnacionais e suas redes horizontais na construção da governança ambiental global.

\subsection{Novos atores da sociedade internacional e a paradiplomacia ambiental}

A participação de representantes do setor privado, de ONGs e de governos subnacionais nos processos de negociação multilaterais motivou a ampliação do debate internacional para novos atores que vão além dos Estados nacionais. ${ }^{26},{ }^{27} \mathrm{~A}$ inserção desses novos atores na sociedade internacional está diretamente associada a dois fenômenos marcantes do século XX: o processo de globalização e a emergência de problemas ambientais globais, como as mudanças climáticas e a proteção da biodiversidade.

${ }^{25}$ GONÇALVES, Alcindo; FONTOURA COSTA, José Augusto. Governança global e regimes internacionais. São Paulo: Almedina, 2010.

${ }^{26}$ KEOHANE, Robert; NYE, Joseph (Eds.). Transnational relations and world politics. Cambridge: Harvard University Press, 1971.

${ }^{27}$ RISSE-KAPPEN, Thomas (Ed). Bringing transnational relations back. In: NON-STATE actors, domestic structures and international institutions. Cambridge: Cambridge University Press, 1995. 
Em primeiro lugar, há um processo de globalização que se caracteriza pela ruptura das fronteiras dos meios de comunicação e de informação, de transporte e de distribuição, assim como da produção e do consumo. ${ }^{28}$ Ao mesmo tempo em que intensifica a interdependência global, a globalização acaba por relativizar o papel do Estado-Nação como único interlocutor legítimo das relações internacionais. Governos subnacionais, empresas, organizações não governamentais e até indivíduos passam a transcender os mecanismos verticalizados de articulação internacional, formando espaços horizontais de interação em que podem, de forma mais direta e dinâmica, exercer seus interesses concretos.

Em segundo lugar, a evolução da ciência do clima tem reforçado o caráter universal das mudanças climáticas, seja porque suas causas antrópicas estão no cerne do atual modo de produção e consumo no planeta, seja porque a gravidade de seus impactos será sentida em todos os níveis da sociedade - do local ao global -, e sob as diferentes nuances - ambientais, sociais, econômicas e políticas. ${ }^{29}$ Daí porque o seu enfrentamento revela-se um desafio. Daí também, porque as ações práticas de enfrentamento dos problemas ambientais globais têm progressivamente legitimado o surgimento de novas formas de autoridade, as quais ganham uma legitimação voluntária da sociedade ainda que desprovidas dos elementos da soberania, autonomia e controle. ${ }^{30}$

A inserção dos governos subnacionais no enfrentamento das mudanças climáticas é uma das facetas dessa nova forma de governança ambiental global. Em meados da década de 1980, a inserção dos governos subnacionais na esfera internacional, principalmente em questões de cunho econômico, recebeu o nome de

\footnotetext{
${ }^{28}$ BEDIN, Gilmar Antonio. A sociedade global e suas possibilidades de realização: um olhar a partir das relações internacionais. In: OLIVEIRA, Odete Maria; DAL RI JR., Arno (Org.). Relações internacionais: interdependência e sociedade global. Ijuí: Editora Unijuí, 2003. p.505-536.

${ }^{29}$ MATEO, Ramón Martin. Tratado de derecho ambiental. Madrid: Trivium, 1992. v.2.

${ }^{30}$ DEDUERWAERDERE, Tom. The contribution of network governance to sustainable development. Belgique: Université Catholique de Louvain Fonds National de la Recherche Scientifique, 2005.
}

"paradiplomacia".31, 32, 33, 34,35 Mais recentemente, o conceito de "paradiplomacia" passou a ser utilizado também para expressar o crescente interesse e participação direta de atores subnacionais nas questões ambientais globais, bem como na formulação e na implementação da politica climática. ${ }^{36},{ }^{37},{ }^{38}$ Neste artigo, o termo "paradiplomacia ambiental" refere-se à inserção dos governos subnacionais no DIMA, isto é, a "paradiplomacia ambiental" diz respeito às iniciativas transnacionais estabelecidas por uma região ou por um estado subnacional para combater problemas ambientais globais. Tais iniciativas incluem a assinatura de acordos e parcerias com Estados e regiões de países estrangeiros, a participação em conferências e eventos internacionais, assim como a participação desses entes em redes transnacionais de governos subnacionais.

$\mathrm{Na}$ literatura, são identificados diferentes vetores desse movimento para além das fronteiras do país no qual se insere um determinado estado subnacional. Para Bueno da Silva, o engajamento dos governos subnacionais em alianças transnacionais decorre da percepção sobre custos e benefícios de participar em determinadas ações

${ }^{31}$ DUCHACEK, Ivo. The international dimensions of subnational self-government. Publius, v. 14, n. 4, p. 5-31, 1984.

32 MICHELMANN, Hans J.; SOLDATOS, Panayotis. (Eds.). Federalism and international relations: the role of subnational units. Oxford: Oxford University Press, 1990.

${ }^{33}$ KEATING, Michael. Regions and international affairs: motives, opportunities and strategies. In: ALDECOA, Francisco; KEATING, Michael. Paradiplomacy in action: the foreign relations of subnational governments. London: Frank Cass Publishers, 1999. p.231.

${ }^{34}$ LECOURS, Andre. Paradiplomacy: reflections on the foreign policy and international relations of regions. International Negotiation, v. 7, p. 91-114, 2002.

${ }^{35}$ HOCKING, Brian. Regionalismo: uma perspectiva das relações internacionais. In: VIGEVANI, Tulio et al. (Org.). A dimensão subnacional e as relações internacionais. São Paulo: EDUC, 2004. p. 77-108.

${ }^{36}$ HOCKING, Brian. The woods and the trees: catalytic diplomacy and Canada's trials as a forestry superpower. Environmental Politics, v. 5, n. 3, p. 448-75, 1996.

${ }^{37}$ SILVA, Ironildes Bueno da. Paradiplomacia contemporânea: trajetórias e tendências da atuação internacional dos governos estaduais do Brasil e EUA. Brasília. 2010. f. ? Tese (Doutorado em Relações Internacionais)-Universidade de Brasília (IREL/UnB), Brasília, 2010.

${ }^{38}$ EATMON, Thomas D. Paradiplomacy and climate change: American States as actors in global climate governance. Journal of Natural Resources Policy Research, v. 1, n. 2, p. 153-165, 2009. 
ou políticas na área ambiental. ${ }^{39}$ Analisando uma série de iniciativas locais e transregionais estadunidenses voltadas ao enfrentamento das mudanças climáticas, Engels e Orbach $^{40}$ identificaram uma correlação direta dessas ações a apelos eleitorais ou de apoio político junto à população, mas nem sempre assentadas em medidas operacionais de enfrentamento. Já para Bernstein et $\mathrm{al},{ }^{41}$ o estado atual da negociação sobre o avanço do regime internacional do clima, marcado pela resistência dos Estados em assumir maiores e novos compromissos, mina a legitimidade dos Estados e reforça outros polos de autoridade e tomada de decisão, em particular os governos subnacionais, que estão no nível imediatamente abaixo do governo central.

No caso dos temas ambientais globais, como já afirmado, a agenda internacional dos estados subnacionais tem o potencial de influenciar direta ou indiretamente as negociações internacionais sobre mudança do clima, atuando como polos de pressão junto aos Estados, para que avancem com seus compromissos e esforços de enfrentamento do problema. Para Hoffmann, uma verdadeira experimentação de governança climática se consolida na Conferência das Partes de Copenhague a partir das ações políticas e técnicas das delegações subnacionais. ${ }^{42}$

Além disso, a participação dos atores subnacionais na governança ambiental global ganha força na medida em que tais atores agem por meio de redes horizontais de cooperação. ${ }^{43},{ }^{44}$. Conforme reconhece o parágrafo 62 do Zero Draft, as parcerias entre cidades têm liderado a

${ }^{39}$ SILVA, Ironildes Bueno da. Paradiplomacia contemporânea: trajetórias e tendências da atuação internacional dos governos estaduais do Brasil e EUA. Brasília. 2010. Tese (Doutorado em Relações Internacionais) - Universidade de Brasília (IREL/UnB), Brasília, 2010.

${ }^{40}$ ENGELS, Kirsten; ORBACH, Barak. Micro-motives and state and local climate change initiatives. Harvard Law and Policy Review, v. 2, p. 119-137, 2008.

${ }^{41}$ BERNSTEIN, Steven et al. A tale of two copenhagens: carbon markets and climate governance. Millennium: Journal of International Studies, v. 39, n. 1, p. 161-173, 2010.

${ }^{42}$ HOFFMANN, Matthew J. Climate governance at the crossroads. New York: Oxford University Press, 2011.

${ }^{43}$ BULKELEY, Harriet, BETSILL, Michelle. Cities and climate change: urban sustainability and global environmental governance. London: Routledge, 2003.

${ }^{44}$ SETZER, Joana. Governança multinível das mudanças climáticas: políticas subnacionais e ações transnacionais em São Paulo. In: REI, Fernando Cardozo Fernandes (Org.). Direito e desenvolvimento: uma abordagem sustentável. No prelo. ação para o desenvolvimento sustentável. ${ }^{45}$ Ademais, a participação dos estados subnacionais nessas redes transnacionais oferece a esses atores o potencial de influenciar a tomada de decisão nos fóruns internacionais, além de lhes conferir uma personalidade internacional. ${ }^{46}$ Apesar de formalmente criadas como mecanismos de troca de informação, técnicas, práticas e experiência em medidas de enfrentamento das mudanças climáticas, essas redes transnacionais acabam por exercer um crescente papel político, principalmente quando atuam de forma coordenada na esfera internacional de negociação. ${ }^{47}$ Esse é o caso da Rede de Governos Regionais para o Desenvolvimento Sustentável (nrg4SD).

\subsection{A Nrg4SD}

Dentre as modalidades da paradiplomacia ambiental, a nrg4SD chama atenção por ser a primeira e única rede transnacional exclusiva para governos subnacionais a tratar do tema do desenvolvimento sustentável em uma escala global. ${ }^{48}$ As negociações iniciais objetivando a criação dessa rede de governos regionais aconteceram alguns meses antes da Cúpula Mundial sobre Desenvolvimento Sustentável de Johanesburgo, no âmbito da reunião preparatória de maio de 2002, em Bali. Durante a Cúpula, por meio da Declaração de Gauteng, foi então instituída a nrg4SD, com o objetivo de promover trocas de experiências, conhecimento e colaboração entre os governos subnacionais regionais a respeito de sustentabilidade ambiental. Atualmente, a nrg4SD conta com a

${ }^{45}$ No mesmo parágrafo 62, item D da Seção IV (“Quadro institucional para um desenvolvimento sustentável”), os Estados-Membros comprometem-se, inclusive, por meio da assistência conferida por organizações internacionais, a apoiar a cooperação internacional entre governos subnacionais.

${ }^{46}$ HAPPAERTS, Sander; VAN DEN BRANDE, Karoline; BRUYNINCKX, Hans. Governance for sustainable development at the inter-subnational level: the case of the network of regional governments for sustainable development (nrg4SD). Regional \& Federal Studies, v. 20, n. 1, p. 127-149, 2010.

${ }^{47}$ BULKELEY, Harriet. Reconfiguring environmental governance: towards a politics of scales and networks. Political Geography, n. 24, p. 875-902, 2005.

${ }^{48}$ Mais recentemente, em novembro de 2010, as vésperas da COP16 em Cancun, foi criada a R20 Regions of Climate Action. A R20 foi concebida pelo então Governador da Califórnia, Arnold Schwarzenegger. Mas além de governos subnacionais, a R20 tem também membros não governamentais e privados (R20, 2011). Happaerts, Brande e Bruyninckx, também chamam atenção para o fato de que, diferentemente de outras redes de governos subnacionais, a nrg $4 \mathrm{SD}$ possui um escopo Norte-Sul e um foco nos organismos das Nações Unidas. 
participação de 36 governos subnacionais de 18 países e 7 associações de governos regionais, como membros plenos, que, ao todo, representam mais de 1000 governos em todo o mundo.

Vê-se que ao longo de seus dez anos de existência, a nrg4SD passou de uma iniciativa basicamente ad hoc para uma organização consolidada que possui uma estrutura definida, além de caráter e procedimentos permanentes. ${ }^{49}$ Em 2004, a nrg4SD obteve a qualificação jurídica de associação civil sem fins lucrativos, de cunho internacional, segundo a legislação da Bélgica, e mantém em Bruxelas a sua sede. A coordenação das atividades da nrg4SD é feita por um Comitê Executivo, que se reúne duas vezes ao ano para estabelecer as diretrizes gerais de atuação. Esse comitê é composto por membros plenos eleitos pelo período de três anos. ${ }^{50}$

Desde a primeira grande conferência realizada em San Sebastian, no País Basco, em março de 2003, a Rede realizou e participou de mais de uma centena de conferências e eventos internacionais, levando o exemplo das regiões para a agenda internacional da sustentabilidade. Em virtude das tipologias admitidas pelo sistema das $\mathrm{Na}$ ções Unidas, a nrg4SD foi credenciada como um observador da sociedade civil perante UNFCCC. Além disso, a rede mantém uma força-tarefa para as negociações internacionais sobre mudança do clima, e um grupo de trabalho sobre financiamento em mudança desta mudança no nível subnacional. Em três ocasiões, a nrg4SD promoveu Cimeiras Mundiais das Regiões, com a participação de dezenas de mandatários de governos regionais, permitindo a troca de experiências, o contato pessoal desses representantes para a construção de agendas políticas a partir de desafios ambientais. ${ }^{51}$

\footnotetext{
${ }^{49}$ HAPPAERTS, Sander; VAN DEN BRANDE, Karoline; BRUYNINCKX, Hans. Governance for sustainable development at the inter-subnational level: the case of the network of regional governments for sustainable development (nrg4SD). Regional \& Federal Studies, v. 20, n. 1, p. 127-149, 2010.

${ }^{50}$ NRG4SD. Disponível em: <http://www.nr4sd.org/>. Acesso em: 17 mar. 2012.

${ }^{51}$ A primeira Cimeira teve lugar em Montreal, em dezembro de 2005; a segunda, em Saint Malo, Bretanha, em outubro de 2008 e a última, em Montevidéu, em abril de 2010. Durante a Cimeira de Saint Malo foi assinado um acordo entre o PNUD, o PNUMA e as regiões, que marcou o ponto de partida de um processo político no qual as regiões do mundo, lideradas pela nrg4SD, passaram a associar-se mais estreitamente com a ação global contra as mudanças climáticas.
}

Além disso, a nrg4SD fomenta projetos de cooperação Norte-Norte, Norte-Sul e Sul-Sul e utiliza mecanismos de participação direta nas discussões internacionais para advogar pelo reconhecimento da contribuição dos governos subnacionais para o desenvolvimento sustentável (nrg4SD, 2011b). Para fortalecer sua participação na arena internacional, a nrg4SD atua de forma cooperada com outras redes de governos subnacionais e ONGs, com destaque para o Fórum Global de Regiões (FOGAR), o The Climate Group (TCG), os ICLEI Governos Locais para a Sustentabilidade (ICLEI) e Cidades Unidas e Governos Locais (UCLG).

Como resultado de suas atividades nos "corredores" das negociações internacionais, a nrg4SD tem influenciado a comunidade internacional a reconhecer o papel dos Estados subnacionais no âmbito de importantes acordos multilaterais ambientais. $\mathrm{Na}$ reunião da Convenção de Diversidade Biológica (CDB), ocorrida em outubro de 2010, em Nagoya, as partes adotaram a Decisão X/22, pela qual foi ratificado o Plano de Ação de Governos Subnacionais, Cidades e outras Autoridades Locais. ${ }^{52}$ Um mês depois, na COP do Clima, em Cancun, a nrg4SD conseguiu junto aos delegados dos Estados a inclusão do texto sobre o papel dos governos subnacionais, reconhecendo a sua importância no enfrentamento das mudanças climáticas. ${ }^{53}$ Esses andamentos sugerem que os governos subnacionais estão se inserindo como novos atores no processo internacional de tomada de decisão na área do desenvolvimento sustentável. ${ }^{54}$

${ }^{52}$ O Plano de Ação de Governos Subnacionais, Cidades e outras Autoridades Locais reforça a necessidade de incluir estes níveis de governo na implantação das medidas de proteção da biodiversidade acordadas em Nagoya, bem como reconhece os esforços regionais e locais como ações implementadas no âmbito da CDB.

${ }^{53}$ A Decisão 1/CP16 dos Acordos de Cancun, firmados na COP 16 , formalmente reconhece: "[...] the need to engage a broad range of stakeholders at the global, regional, national and local levels, be they government, including subnational and local government, private business or civil society, including youth and persons with disability, and that gender equality and the effective participation of women and indigenous peoples are important for effective action on all aspects of climate change" (UNFCCC, 2011).

${ }^{54}$ HAPPAERTS, Sander; VAN DEN BRANDE, Karoline; BRUYNINCKX, Hans. Governance for sustainable development at the inter-subnational level: the case of the network of regional governments for sustainable development (nrg4SD). Regional \& Federal Studies, v 20, n. 1, p. 127-149, 2010. 


\subsection{A posição da nrg4sd sobre o zero draft da rio +20}

Em janeiro de 2012, a nrg4SD posicionou-se sobre o Zero Draft, formulando quatro pedidos aos Estados-Membros: promoção do PNUMA a uma agência especializada com representação universal e recursos adequados; transformação da Comissão de Desenvolvimento Sustentável (UNCSD) em um Conselho vinculado à Assembleia Geral; introdução de metas de desenvolvimento sustentável com indicadores e metas claras; e criação de conselhos nacionais de desenvolvimento sustentável.

Especificamente com relação à referência feita pelo Zero Draft às autoridades locais e à dimensão local, a nrg4SD se manifestou no sentido de que existe margem para melhorar a integração dos governos locais e subnacionais nos processos de tomada de decisão e implementação dos compromissos assumidos. ${ }^{55}$ Basicamente, a rede de governos regionais entende que:

The Zero Draft published on 9 January 2012 contains important mentions to the Major Group 'Local Authorities' and the local dimension, acknowledging the essential role of local governments and the need to fully integrate them into all levels of decision making on sustainable development (paragraph 20). The wording of the Zero Draft, however, is not fully satisfactory to intermediate levels of governments - such as Federated States, Regions and Provinces - because all subnational actors are subsumed under the expression 'local authorities. ${ }^{56}$

Durante as discussões iniciais do Zero Draft realizadas entre 25 e 27 de janeiro de 2012, em Nova Iorque, diversas propostas para a inclusão do termo 'subnacional' foram feitas especialmente pelo Canadá, pela União Europeia, pela Coreia e pela Suíça. A nrg4SD contribuiu para essas discussões por meio do Grupo Principal de Autoridades Locais. O texto apresentado na sessão do dia 27 de janeiro dá sinais de reconhecer a importância dos 'governos subnacionais', de forma distinta das 'autoridades locais' para o desenvolvimento sustentável.

${ }_{55}$ CARDAMA, Maruxa. Rio +20 Zero Draft: Comment on the initial discussions. Disponível em: <http:// www.stake holderforum .org/sf/outreach /index.php / outreach2012zero3home/627-outreach2012zero3tem13>. Acesso em: 27 jan. 2012.

${ }^{56}$ NRG4SD. Briefing note da nrg4SD sobre o Zero Draft e discussões iniciais na ONU. Disponível em: $<$ http://www.nrg4sd.org /sites/default/files/default /files/content /public/32sustdev/ rio/briefing_note_on_zero_draft_and_initial_discussions_ jan.pdf>. Acesso em: 11 mar. 2012.
Até o início da Conferência, no dia 20 de junho de 2012, a nrg4SD e o Grupo Principal de Autoridades Locais terão uma agenda movimentada, que inclui a participação no encontro de Grupos Principais em Nairobi, Quênia (18 e 19 de fevereiro), na primeira rodada informal de negociações do Zero Draft (19 a 23 de março), nas reuniões da UNCSD (26 e 27 de março e 13 a 15 de junho) e do Encontro Mundial de Estados e Regiões (19 de junho). ${ }^{57}$ Em suma, o posicionamento da rede e a mobilização que se observa no âmbito do Grupo Principal de Autoridades Locais da Rio+20 indicam como os governos subnacionais têm se empenhado para serem reconhecidos como stakeholders governamentais no conjunto de atores que integra o cenário internacional de tomada de decisão.

\section{Conclusão e recomendações}

Este artigo demonstrou que os problemas que afetam o mundo hoje exigem uma resposta de múltiplos atores, em múltiplas escalas, cabendo aos governos subnacionais o papel crucial de desenvolver e implementar as políticas necessárias à promoção do paradigma da sustentabilidade. Ou seja, não será possível atingir o desenvolvimento sustentável almejado pela Rio+20 sem a direta participação dos governos subnacionais na implementação de políticas públicas.

Mais do que isso, o artigo demonstrou que não se trata apenas de reconhecer a importância dos governos subnacionais como atores responsáveis pela implementação das políticas necessárias ao desenvolvimento sustentável. Fazendo uso da paradiplomacia ambiental, os governos subnacionais buscam um espaço para contribuir no processo internacional de tomada de decisão. Em outras palavras, percebe-se como para se alcançar uma verdadeira governança ambiental global os governos subnacionais precisam ser reconhecidos como stakeholders governamentais no conjunto de atores que integra o cenário internacional de tomada de decisão.

Ao longo dos últimos anos, individualmente e com outros parceiros estratégicos, a nrg4SD liderou as regiões do mundo junto a diversas instâncias do sistema

\footnotetext{
${ }^{57}$ A nrg4SD também agendou a reunião do seu Comitê Diretor e sua Assembleia Geral para os dias 17 e 18 de junho.
} 
das Nações Unidas, objetivando o reconhecimento do papel dos governos subnacionais enquanto atores necessários da agenda internacional da sustentabilidade. Esse reconhecimento se deu, em um primeiro momento, no âmbito da Conferência da Biodiversidade, em novembro de 2010 em Nagoya, com a Decisão X/22. O Plano de Ação de Governos Subnacionais, Cidades e outras Autoridades Locais, ratificado naquela ocasião, reforça a necessidade de incluir todos os níveis de governo na implantação das medidas de proteção da biodiversidade, bem como reconhece os esforços regionais e locais como ações implementadas no âmbito da CDB.

O mesmo reconhecimento se deu na Conferência de Cancun, no mês seguinte, no regime internacional das mudanças climáticas. A importância desse regime destaca a emergência e a relevância da paradiplomacia ambiental no âmbito dos Acordos Ambientais Multilaterais, abrindo um espaço sem precedentes para que os governos subnacionais possam contribuir em um novo regime internacional para a governança climática multinível, sinalizando que se aproximam novos tempos e, quiçá, modelos para os futuros acordos que possam surgir após o primeiro período de compromissos do Protocolo de Quioto.

Com os resultados da COP-17, em Durban, África do Sul, que sinalizaram um singelo avanço nas negociações diplomáticas e na consecução de metas mandatórias para um novo período de compromissos, os governos regionais do mundo se fortalecem para mostrar ao mundo em 2012, com a realização da Rio+20 e da COP-18, no Qatar, que, com transparência, cooperação técnica e com o envolvimento participativo de regiões desenvolvidas e em desenvolvimento em pequenos grupos de trabalho temáticos, a paradiplomacia pode ser uma alternativa dinâmica para o enfrentamento com resultados dos complexos problemas ambientais do século XXI. O processo da Rio+20 oferece, nesse sentido, um espaço importante para os governos subnacionais. Além de reconhecer a governança em todos os níveis de governo como parte integrante do quadro institucional do desenvolvimento sustentável (como já consta no Zero Draft), o processo da Rio+20 poderá vir a confirmar o papel dos governos subnacionais como stakeholders governamentais no conjunto de atores que integra o cenário internacional de tomada de decisão.

\section{Referências}

ADGER, Neil; JORDAN, Andrew (Ed.). Governing sustainability. Cambridge: Cambridge University Press, 2009.

BEDIN, Gilmar Antonio. A sociedade global e suas possibilidades de realização: um olhar a partir das relações internacionais. In: OLIVEIRA, Odete Maria; DAL RI JR., Arno (Org.). Relações internacionais: interdependência e sociedade global. Ijuí: Unijuí, 2003. p. 505-536.

BERNSTEIN, Steven et al. A tale of two Copenhagens: carbon markets and climate governance. Millennium: Journal of International Studies, v. 39, n. 1, p. 161-173, 2010 .

BODANSKY, Daniel. The legitimacy of international governance: a coming challenge for international environmental law? The American Journal of International Law, v. 93, n. 3, p. 596-624, jul.1999.

BULKELEY, Harriet. Reconfiguring environmental governance: towards a politics of scales and networks. Political Geography, n. 24, p. 875-902, 2005.

BULKELEY, Harriet; BETSILL, Michelle. Cities and climate change: urban sustainability and global environmental governance. London: Routledge, 2003.

CARDAMA, Maruxa. Rio+20 Zero Draft: comment on the initial discussions. Disponível em: <http://www.stakehol derforum. org /sf/ outreach/index.php/outreach2012zero3home/627outreach2012zero3tem13>. Acesso em: 27 jan. 2012.

DEDUERWAERDERE, Tom. The contribution of network governance to sustainable development. Belgique: Université Catholique de Louvain Fonds National de la Recherche Scientifique, 2005.

DUCHACEK, Ivo. The international dimensions of subnational self-government. Publius, v. 14, n. 4, p. 5-31, 1984.

EATMON, Thomas D. Paradiplomacy and climate change: american states as actors in global climate governance. Journal of Natural Resources Policy Research, v. 1, n. 2, p. 153-165, 2009.

ENGELS, Kirsten; ORBACH, Barak. Micro-motives and state and local climate change initiatives. Harvard Law and Policy Review, v. 2, p. 119-137, 2008.

GONÇALVES, Alcindo; FONTOURA COSTA, José Augusto. Governança global e regimes internacionais. São Paulo: Almedina, 2010. 
HAPPAERTS, Sander; VAN DEN BRANDE, Karoline; BRUYNINCKX, Hans. Governance for sustainable development at the inter-subnational level: the case of the network of regional governments for sustainable development (nrg4SD). Regional \& Federal Studies, v. 20, n. 1, p. 127-149, 2010.

HOCKING, Brian. Regionalismo: uma perspectiva das relações internacionais. In: VIGEVANI, Tulio; WANDERLEY, Luiz Eduardo; BARRETO, Maria Ines; MARIANO, Marcelo Passini (Org.). A dimensão subnacional $e$ as relações internacionais. São Paulo: EDUC; Fundação Editora da UNESP, 2004. p. 77-108.

HOCKING, Brian. The woods and the trees: catalytic diplomacy and Canada's trials as a forestry superpower. Environmental Politics, v.5, n.3, p. 448-75, 1996.

HOFFMANN, Matthew J. Climate governance at the crossroads. New York: Oxford University Press, 2011.

KEATING, Michael. Regions and international affairs: motives, opportunities and strategies. In: ALDECOA, Francisco; KEATING, Michael. Paradiplomacy in action: the foreign relations of subnational governments. London: Frank Cass Publishers, 1999. p. 231.

KEOHANE, Robert, NYE, Joseph (Eds.). Transnational relations and world politics. Cambridge: Harvard University Press, 1971.

KRAHMAN, Elke. National, regional and global governance: one phenomenon or many. Global governance, v. 9, p. 323-346, 2003.

LECOURS, Andre. Paradiplomacy: reflections on the foreign policy and international relations of regions. International negotiation, v. 7, p. 91-114, 2002.

LEIS, Hector; VIOLA, Eduardo. América del Sur en el mundo de las democracias de mercado. Rosario: Homo Sapiens Ediciones, 2008. p. 196.

LITFIN, Karen. Environment, Wealth and Authority: global climate change and emerging modes of legitimation. International studies review, v. 2, n. 2, p. 119-148, 2002.

MATEO, Ramón Martin. Tratado de derecho ambiental. Madrid: Trivium, 1992. v.2.

MICHELMANN, Hans J.; SOLDATOS, Panayotis. (Eds.). Federalism and international relations: the role of subnational units. Oxford: Oxford University Press, 1990.

NRG4SD. Disponível em <http://www.nr4sd.org/>. Acesso em: 17 mar. 2012.
NRG4SD. Briefing note da nrg4SD sobre o Zero Draft e discussões iniciais na ONU. Disponível em: <http://www. nrg4sd.org/ sites/default/f iles/default/ files/content/ public/32-sustdev/rio/briefing_note_on_zero_draft_ and_initial_discussions_jan.pdf $>$. Acesso em: 11 mar. 2012.

OKEREKE, Chukwumerije; BULKELEY, Harriet; SCHROEDER, Heike. Conceptualizing climate governance beyond the international regime. Global environmental politics, v. 9, n. 1, p. 58-68, fev. 2009.

OSOFSKY, Hari .M. Multiscalar Governance and climate change: reflections on the role of states and cities at Copenhagen. Maryland Journal of International Law, v. 25, n. 64, p. 64-85, 2010.

RABE, Barry. States on steroids: the intergovernmental odyssey of american climate policy. Review of Policy Research, v. 25, n. 2, p. 105-128, 2008.

RABE, Barry. Statehouse and greenhouse: the emerging politics of american climate change policy. Washington, D.C.: Brookings Institution Press, 2004.

REED, Maureen; BRUYNEEL, Shannon. Rescaling environmental governance, rethinking the state: a threedimensional review. Progress in Human Geography, v. 34, n. 5, p. 646-653, 2010.

REI, Fernando, CUNHA, Kamyla. Mudanças climáticas globais: desafio a uma nova relação entre o direito internacional do meio ambiente e as relações internacionais. In: CASELLA, Paulo Borba et al. (Orgs.). Direito internacional, humanismo e globalidade: guido Fernando Silva Soares Amicorum Discipulorum Líber. São Paulo: Atlas, 2008. p. 487-502.

RISSE-KAPPEN, Thomas (Ed.). Bringing transnational relations back. In: Non-state actors, domestic structures and international institutions. Cambridge: Cambridge University Press, 1995.

RUIZ, Jose Juste. Derecho internacional del medio ambiente. Madrid: McGraw Hill, 1999. p. 479.

SETZER, Joana. Governança multinível das mudanças climáticas: políticas subnacionais e ações transnacionais em São Paulo. In: REI, Fernando Cardozo Fernandes (Org.). Direito e desenvolvimento: uma abordagem sustentável. No prelo.

SILVA, Ironildes Bueno da. Paradiplomacia contemporânea: trajetórias e tendências da atuação internacional dos governos estaduais do Brasil e EUA. Brasília. 2010. Tese (Doutorado em Relações Internacionais) - Universidade de Brasília (IREL/UnB), Brasília, 2010. 
UN. The future we want: zero draft of the outcome document. Disponível em: <http://www. uncsd 2012 . org / rio20 /futurewewant.html>. Acesso em: 11 mar. 2012. 